\title{
SUR DEUX ECHINOGAMMARUS LIMNIQUES NOUVEAUX DU BASSIN D'AQUITAINE
}

\author{
par \\ S. PINKSTER \& J. H. STOCK \\ Institut de Zoologie taxonomique (Zoölogisch Museum), Université d'Amsterdam, Pays-Bas
}

\begin{abstract}
Description of Echinogammarus spinulicornis nov. spec., closely related to $E$. pungens (H. Milne Edwards), and of Echinogammarus zebrinus nov. spec., a member of the $E$. berilloni-group, from streams in the Basin of Aquitaine, southwestern France.
\end{abstract}

L'échantillonnage systématique des eaux superficielles, douces, saumâtres et marines, entrepris pendant 5 ou 6 ans par notre groupe de recherche dans une grande partie de la France, a abouti non seulement à plusieurs notes faunistiques et écologiques (p.e. Stock et al., 1966; Kant, de Leeuw et al., 1968; Pinkster et al., sous presse), mais nous a fait connaître encore de nombreuses espèces de Gammaridae, soit nouvelles pour la faune française (p.e. Stock, 1967; Stock, 1968; Kant, Pinkster \& Stock, 1968), soit nouvelles pour la science. Dans la présente note, nous voulons décrire deux nouvelles espèces limniques, ramassées dans le Bassin d'Aquitaine. Ces deux espèces appartiennent au genre Echinogammarus, l'une au groupe berilloni, l'autre au groupe pungens.

Nous tenons à remercier le personnel scientifique et technique de l'Institut de Biologie marine d'Arcachon pour son hospitalité lors de notre séjour du printemps 1970, la Station servant alors de base pour nos prospections. Une vingtaine d'étudiants de l'Université d'Amsterdam nous ont assistés sur le terrain, et au laboratoire, pour les analyses hydrologiques.

Echinogammarus spinulicornis nov. spec. Figs. 1-3.

Matériel examiné. -

France, dép. Charente: Sources de la Touvre, le Bouillant (près de Ruelle, à l'est d'Angoulême); sous cailloux argileux et entre végétation de Sium; 21 mars 1968, 25 avril 1968 et 14 mai 1970; nombre d'animaux capturés: une centaine d'individus. Eau pure; analyse (mai 1970): température $12^{\circ} 0 \mathrm{C}$, Ca $81 \mathrm{mg} / 1$, CI $18 \mathrm{mg} / 1, \mathrm{pH} 5,5$. Un mâle de cette localité a été choisi comme holotype (ZMA Amph. 102.547).

France, dép. Lot-et-Garone: dans le Gers, près du pont, sur la route départementale 17 à Layrac; largeur de la rivière $8 \mathrm{~m}$ environ, profondeur $1 \mathrm{~m}$ environ; courant fort; 26 avril $1968-1$ \%; 2 mai $1970-13$ spécimens. Eau légèrement polluée. Analyse (mai 1970): température $13^{\circ} 0 \mathrm{C}, \mathrm{Ca} 82 \mathrm{mg} / 1, \mathrm{Cl} 30 \mathrm{mg} / 1, \mathrm{pH} 6,0$.

France, dép. Gers: dans le Gers près du pont sur la route nationale 653 à Fleurance, 22 août 1968; chlorinité $27 \mathrm{mg} / \mathrm{l}, 1$ \%.

Espèces accompagnatrices. - A Ruelle et à Fleurance, la nouvelle espèce était accompagnée d'Echinogammarus berilloni et de Gammarus pulex; à Layrac d' $E$. berilloni et d'E. pacaudi. $E$. spinulicornis n'est pas particulièrement abondant dans ces trois stations, où il ne forme qu'une minorité parmi les populations d'autres Gammares trouvées au même endroit.

Description. - Mâle: Longueur maximale (marge frontale de la tête jusqu' à l'extrémité du 3ème uropode) $13 \mathrm{~mm}$. Corps (planche I fig. 1) assez compact; troisième segment du pléosome dorsalement bombé; premier segment de l'urosome avec forte excavation dorsale ("selle"), suivie d'une élévation latéralement comprimée; deuxième segment de l'urosome avec également une élévation comprimée; dos, à l'exception de l'urosome, glabre (fig. 1G). Les épimères 1 à 3 portent de longues et nombreuses soies sur leur bord ventral. L'angle postérieur de la plaque épimérale 2 est aigu, de la plaque 3 , presque droit.

Oeil, comparé à celui d'autres espèces de Gammares d'eau douce, grand, pourvu d'une pigmentation foncée (fig. 1A). Lobes latéraux de la tête plus ou moins arrondis. 

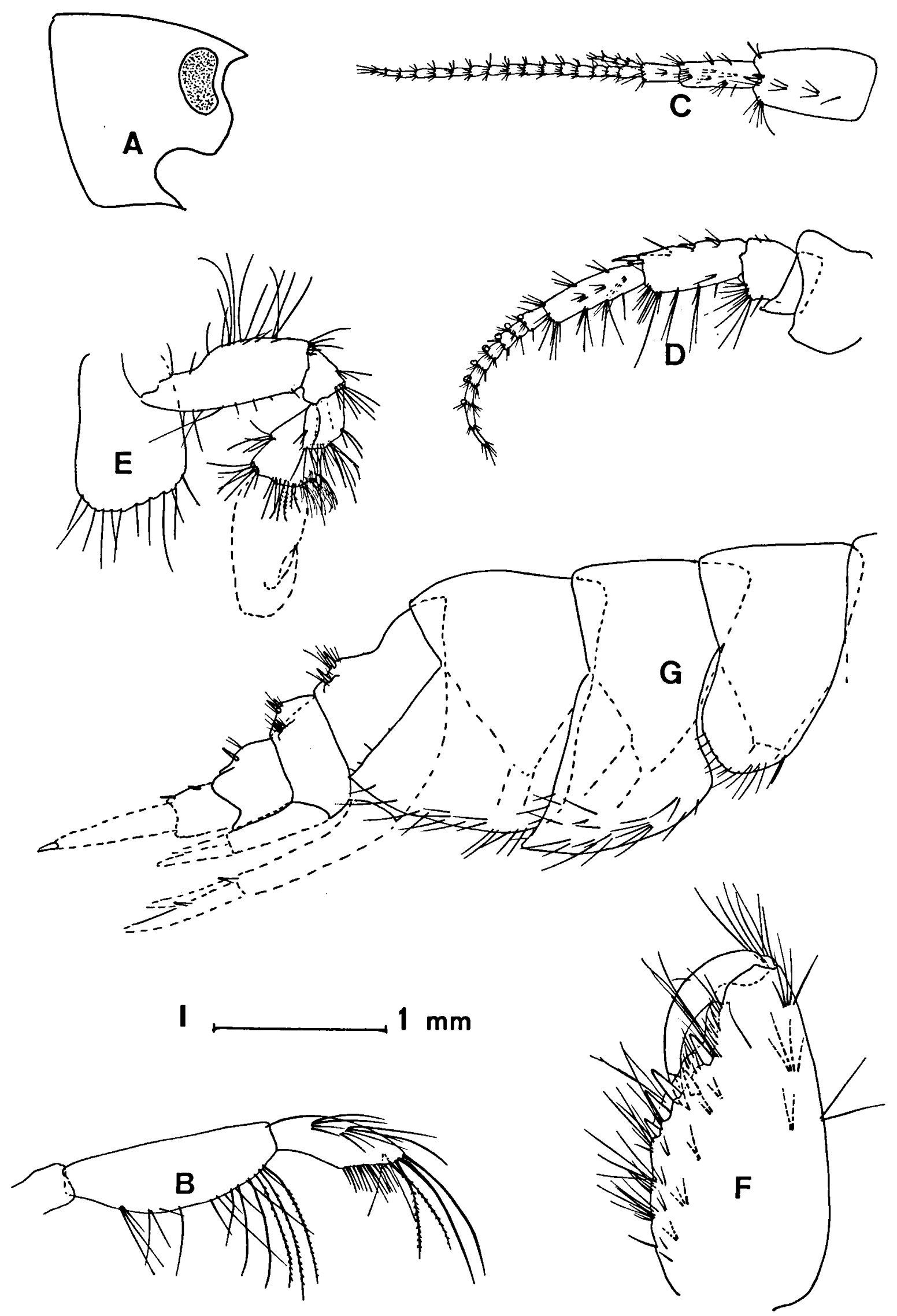

Fig. 1. Echinogammarus spinulicornis nov. spec., $\delta$, paratype, de la Source de la Touvre. A, segment céphalique (échelle I); B, palpe mandibulaire (II); C, antenne supérieure (I); D, antenne inférieure (I); E, première patte (I); F. propode de la première patte (II); G, pléosome et urosome, vue de droite (I) . 
L'antenne supérieure (fig. 1C) est très raccourcie; son flagellum principal se compose de 13 à 16 articles; son flagellum accessoire de 3 à 4 articles. Le pédoncule, pourvu de poils peu nombreux et courts, est formé de trois articles, dont le troisième surtout est très court. Poils du flagellum peu nombreux et très courts.

L'antenne inférieure (fig. 1D) possède un cône glandulaire bref sur le deuxième article du pédoncule. Le 4ème article du pédoncule est très caractéristique par la présence, sur la face dorsale, de 4 épines (dont 2 terminales) au lieu des soies qu'on trouve à cet endroit chez les autres membres du groupe pungens. La face ventrale des articles 4 et 5 ne portent que 4 groupes (dont un terminal) de soies, ni très densement implantées ni très longues. Le flagellum de l'A 2 se compose d'un petit nombre d'articles, à savoir de 7 à 9 ; les 5 à 7 articles proximaux sont pourvus de calcéoles.

Le palpe mandibulaire (fig. 1B) ressemble à celui d'Echinogammarus pungens s. str. (voir fig. 2C), abstraction faite de la longueur légèrement inférieure du 3ème article et du ,peigne” ventral de cet article qui se compose d'un nombre plus petit de soies spiniformes et qui est par conséquent moins long que celui d'E. pungens.

Les pattes 1 à 4 (figs. $1 \mathrm{E}, \mathrm{F} ; 2 \mathrm{~A}, \mathrm{~B}, \mathrm{D}, \mathrm{E}$ ) sont à peu près identiques à celles d' $E$. pungens. Les pattes 5 à 7 (figs. $3 \mathrm{~A}, \mathrm{~B}, \mathrm{C}$ ) ressemblent à celles d'E. pungens par la présence, non seulement sur le bord postérieur mais aussi sur la face interne de l'article basal, de nombreuses soies longues, implantées d'une manière très dense. D'autre part, ces articles basaux sont nettement plus étroits et plus allongés que chez l'autre espèce.

Le telson (fig. 3E) ne montre pas de particularités comparé à celui d'E. pungens. L'endopodite des 3èmes uropodes (fig. 3D) semble légèrement plus allongé que chez $E$. pungens.

Femelle: Longueur maximale (du bord antérieur de la tête jusqu'au bout du 3ème uropode) $9 \mathrm{~mm}$. Pas de calcéoles sur l'A 2, mais des épines dorsales sur le 4ème article pédonculaire. Pas d'épine médiale palmaire sur les gnathopodes 1 et 2 . Les pattes, les épimères et les 3èmes uropodes sont moins poilus que chez le mâle. Le segment basal des pattes 5 à 7 est moins allongé.

Couleur sur le vivant. - D'un gris blanchâtre; intestin, visible par transparence, brun.

Cycle. - L'échantillon de mai 1970 contenait beaucoup de couples précopulés et de femelles ovigères.
Variabilité. - Abstraction faite de la variabilité rencontrée chez tous les Gammares (comme, par exemple, l'augmentation du nombre des articles dans les fouets antennaires selon l'âge), la nouvelle espèce montre une certaine instabilité dans le nombre des poils sur la face dorsale de l'urosome. Les uropodes 3 des plus grands individus sont plus allongées que ceux des moins grands.

Caractères discriminants. - En suivant la clé de détermination des Echinogammarus du groupe pungens, publiée par Stock, $1968: 20$, la présente espèce peut être suivie jusqu'au couplet 13a, $E$. pungens (H. Milne Edwards, 1840), s. str. Elle diffère néanmoins nettement de cette espèce mixohaline, habitant les bords du bassin méditerranéen, par l'ensemble des caractères suivants: (1) le flagellum des $\mathrm{A} 1$ est très raccourci, ne comptant pas plus de 13 à 16 articles (chez pungens on compte de 19 à 27 articles); (2) les épines dorsales du 4ème article pédonculaire de l'A 2 sont très originales, et sont remplacées par des soies chez toutes les autres espèces du groupe; (3) le segment basal des pattes 5 et 6 , mais surtout de la 7ème patte, est plus svelte que chez pungens. Tandis que $E$. pungens habite des eaux salées (estuaires, sources minérales, etc.), $E$. spinulicornis a été trouvé dans des eaux complètement douces, quoique très souvent avec une haute teneur en calcium.

Remarques sur la synonymie et la distribution. -

L'animal décrit ici comme espèce nouvelle, a déjà été signalé des trois localités énumérées cidessus, à savoir par Stock, $1968: 28$, sous le nom provisoire d' "Echinogammarus pungens from outside the Mediterranean drainage system". Les caractères indiqués par Stock, ainsi que ses figures $4 \mathrm{~d}$ et $4 \mathrm{e}$, sont valables pour $E$. spinulicornis, espèce nouvelle basée sur un matériel plus riche ramassé en 1970 sur les localités déjà connues.

La description précédente a été basée sur un matériel ne provenant que de trois stations, toutes situées dans la partie centrale du Bassin d'Aquitaine, à savoir dans les départements de la Charente, du Lot-et-Garonne et du Gers.

La distribution d'Echinogammarus spinulicornis s'étend vers la partie septentrionale de l'Aquitaine, comme l'ont montré Gras \& Maasen (dans le même fascicule de ce périodique), qui le signalent de tout le Pays Charentais. La limite de sa distribution coïncide de façon quasi-total avec la frontière méridionale de la Vendée. La note de Gras \& Maasen donne également quelques précisions 


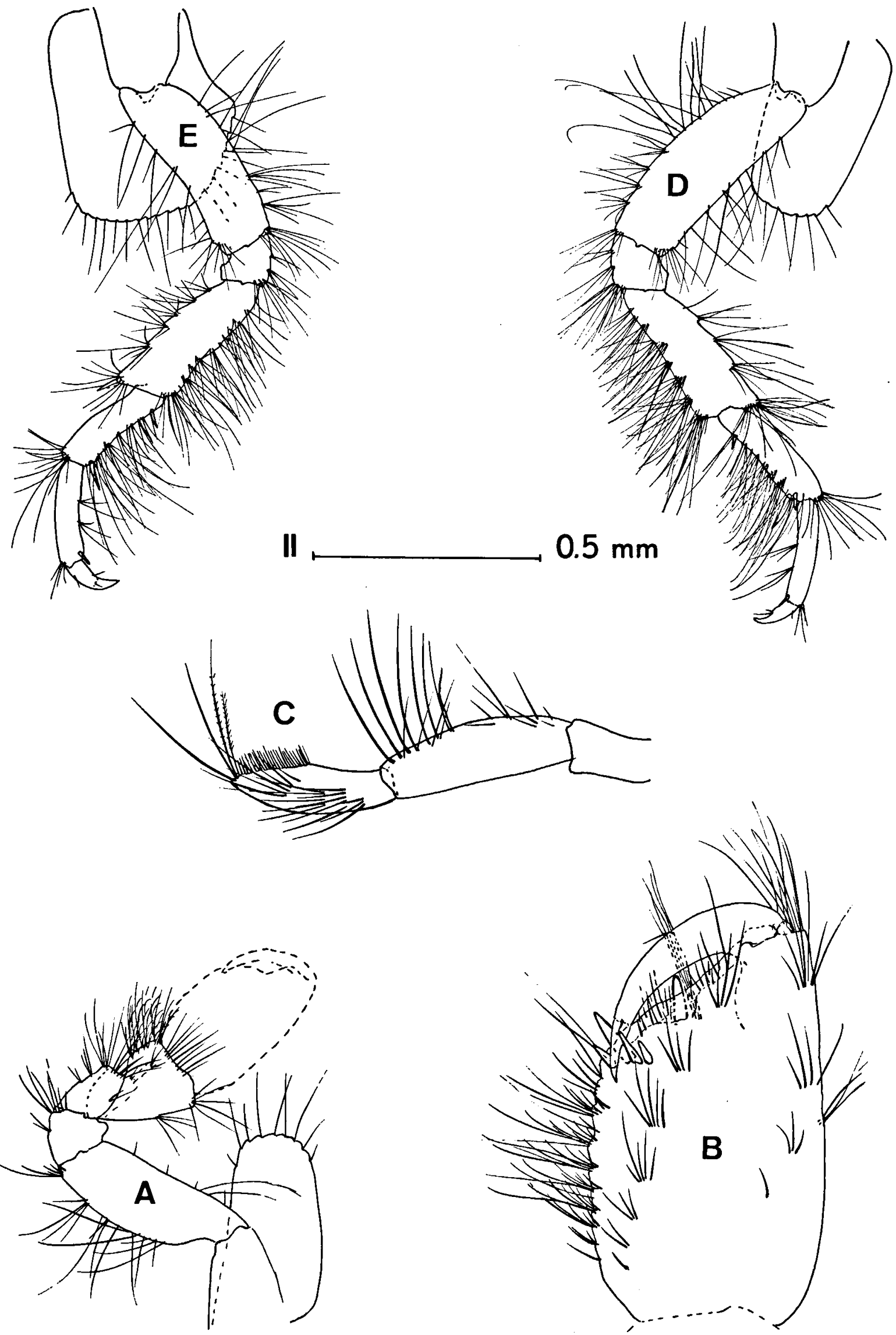

Fig. 2 A, B, D et E. Echinogammarus spinulicornis nov. spec., ô, paratype, de la Source de la Touvre.

Fig. 2 C. Echinogammarus pungens (H. M. Edw.), ô, néotype, de la Fontaine de Salses $_{\star} \mathbf{A}_{\text {; }}$ deuxième patte (échelle $e_{0}: 38 \mathrm{AM}$ I); B, propode de la deuxième patte (II); C, palpe mandi bulaire (II); D, troisième patte (I); E, quatrième pattef (I). access 

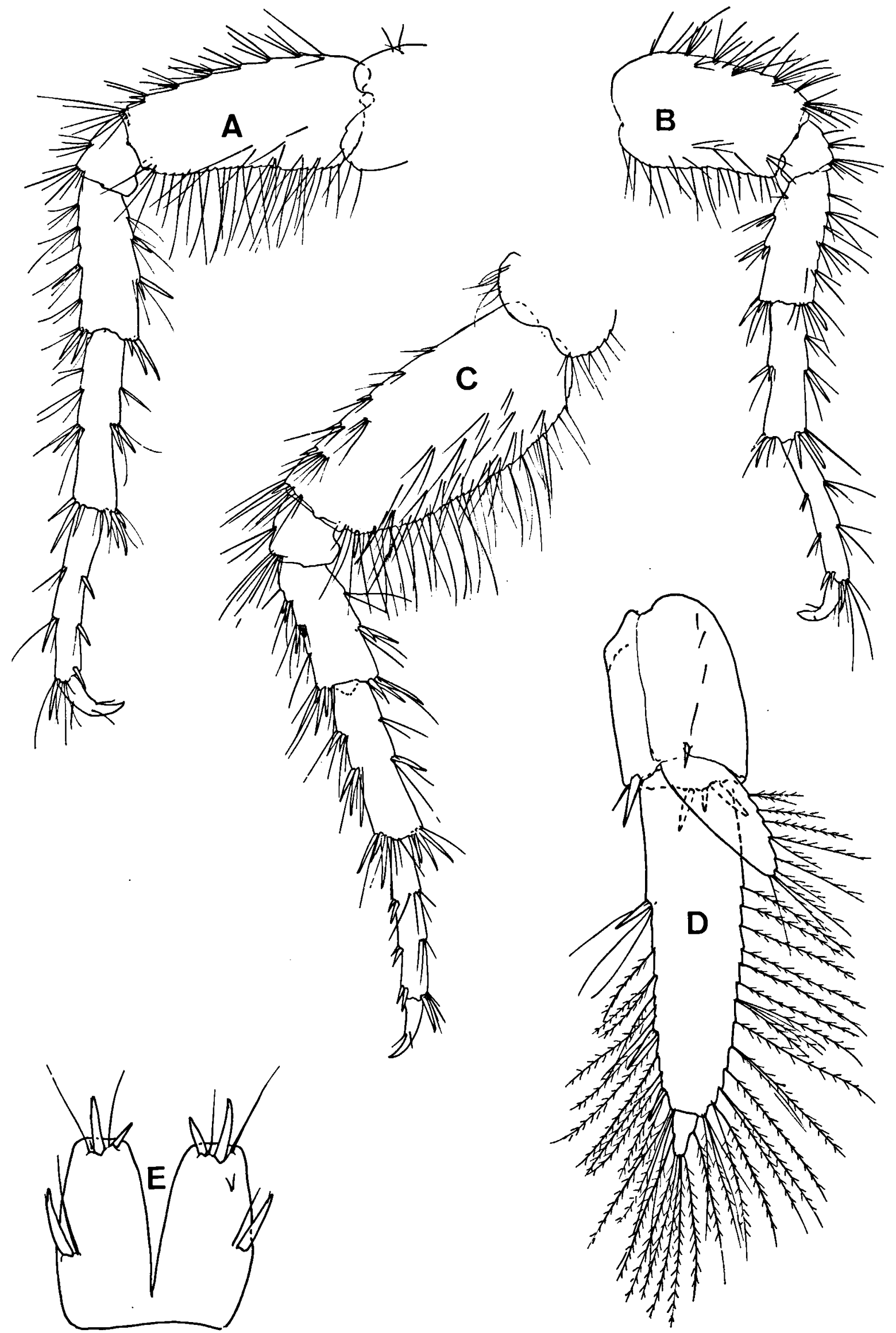

Fig. 3. Echinogammarus spinulicornis nov. spec., A, paratype, de la Source de la Touvre. A, sixième patte (échelle I); B, cinquième patte (I); C, septième patte (I); D, trois ième uropode (II); E, delsone(II). $\circ$ B Brill.come4/26/2023 10:00:38AM 


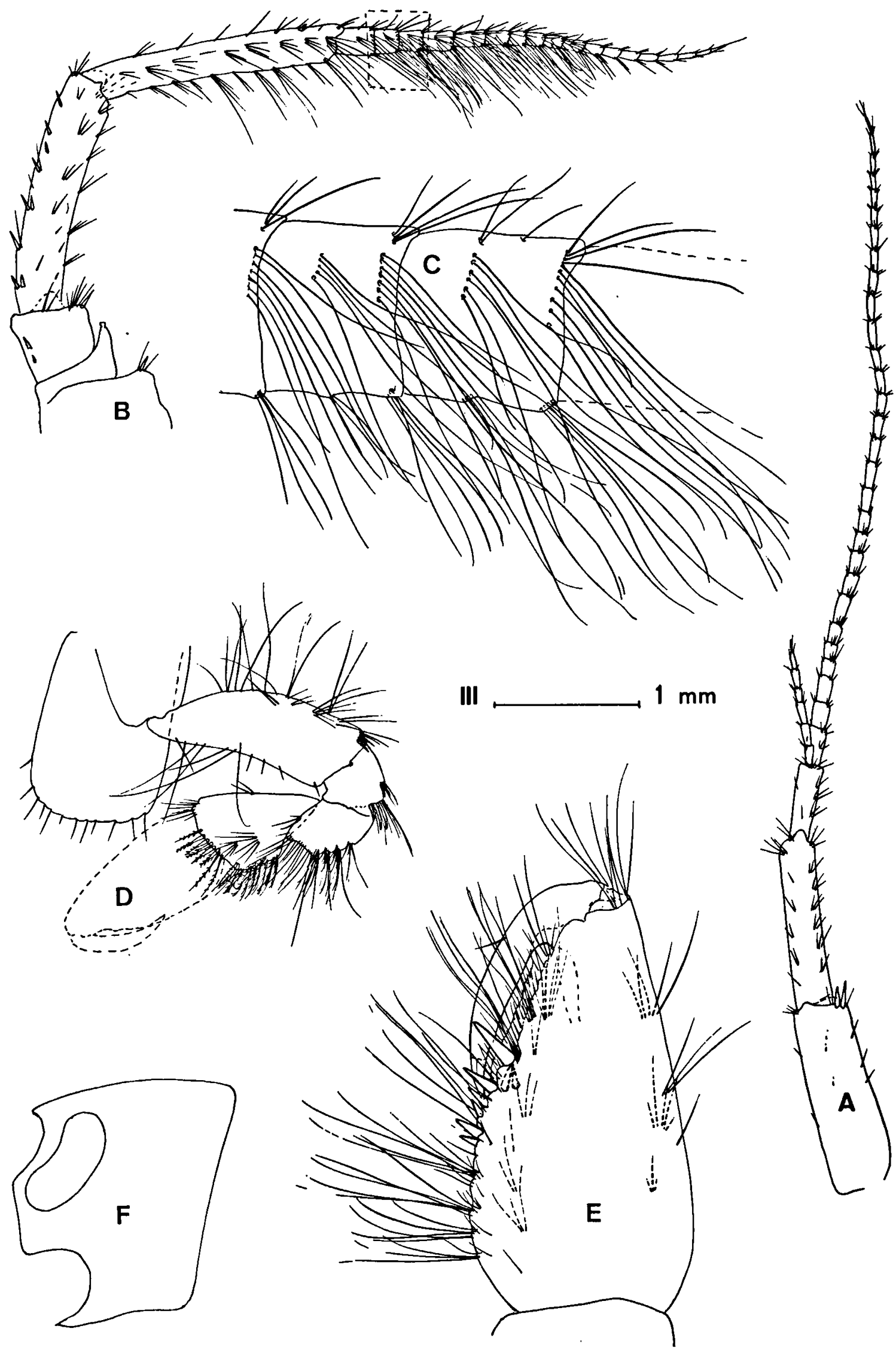

Fig. 4. Echinogammarus zebrinus nov. spec., \&, paratype, de l'Estrigon. A, antenne supérieure (échelle III); B, antenne inférieure (III); C, détail du flagellum de l'antenne inférieure (V); D, première patte (III); E, propode de la pre. mière patte (IV); F, segment céphalique (III). 
concernant les préférences écologiques de l'espèce nouvelle.

Echinogammarus zebrinus nov. spec. Figs. 4-7.

Matériel examiné. -

France, dép. Landes: - Ruisseau l'Estrigon, à Uchacq, au N.O. de Mont-de-Marsan; largeur approximative $10 \mathrm{~m}$, profondeur $1 \mathrm{~m}$; courant faible à modéré; fond sableux; peu de végétation; eau propre; température $12^{\circ} 0 \mathrm{C}$; $\mathrm{Ca}$ $17,9 \mathrm{mg} / \mathrm{l} ; \mathrm{Cl} 24 \mathrm{mg} / \mathrm{l} ; \mathrm{pH} 4,5-5,0 ; 10 \mathrm{mai} \mathrm{1970;78}$ spécimens, parmi lesquels des femelles ovigères et des couples précopulés. Un mâle de cette localité a été choisi comme holotype (ZMA Amph. 102.548). Espèce accompagnatrice: $E$. berilloni.

Ruisseau le Ludon, au nord de St. Gein (= S. de Villeneuve-de-Marsan); largeur 3-4 m; profondeur 30 cm; courant modéré; fond sableux; végétation de Callitriche et d'herbes; eau propre; température $13^{\circ} 9 \mathrm{C}$; $\mathrm{Ca}$ $69,8 \mathrm{mg} / \mathrm{l} ; \mathrm{Cl} 23 \mathrm{mg} / 1$; pH 5,0-5,5; $10 \mathrm{mai} 1970 ; 53$ spécimens, parmi lesquels des femelles ovigères et des couples précopulés. Espèces accompagnatrices: Gammarus pulex, Echinogammarus berilloni, E. pacaudi.

Ruisseau le Midou, près de Villeneuve-de-Marsan; largeur $10 \mathrm{~m}$; profondeur plus d'un mètre; courant faible; fond argileux sans végétation; eau propre; température $12^{\circ} 6 \mathrm{C}$; Ca 53,6 mg/1; Cl $23 \mathrm{mg} / 1$; pH 5,0; $10 \mathrm{mai} 1970$; 32 spécimens, parmi lesquels des femelles ovigères et des couples précopulés. Espèces accompagnatrices: $G$. pulex, $E$. berilloni, E. pacaudi.

Confluent du Midou, $2 \mathrm{~km}$ au sud d'Arthez ( $=$ E. de Villeneuve-de-Marsan); largeur $1 \mathrm{~m}$; profondeur $0,5 \mathrm{~m}$; courant modéré; végétation d'Iris; fond vaseux; eau propre; température $12^{\circ} 8 \mathrm{C}$; Ca $50 \mathrm{mg} / \mathrm{l} ; \mathrm{Cl} 24 \mathrm{mg} / 1$; pH 5,5; 10 mai 1970; 11 spécimens, dont quelques-uns précopulés. Espèces accompagnatrices: $E$. berilloni, $E$. pacaudi.

Confluent du Gouameyre, au nord-est de Cachen (= E. de Roquefort); ruisseau dans une forêt de pins; largeur 5 à $6 \mathrm{~m}$; profondeur $1 \mathrm{~m}$; courant modéré; fond de pierres et de sable; sans végétation; eau propre; température $12^{\circ} 4 \mathrm{C}$; Ca $3,3 \mathrm{mg} / 1 ; \mathrm{Cl} 20 \mathrm{mg} / \mathrm{l} ; \mathrm{pH} \mathrm{5,0;10} \mathrm{mai}$ 1970; 6 spécimens, dont un couple précopulé. Espèce accompagnatrice: $G$. pulex.

Description. - Mâle: Longueur maximale (du bord antérieur de la tête jusqu'à l'extrémité du 3ème uropode) $19 \mathrm{~mm}$. Pléosome et urosome (planche I fig. 2) de contour bossu; pourvus d'épines courbes sur la face dorsale, et de soies, encore courtes et peu nombreuses sur le premier segment du pléosome, mais graduellement plus longues et plus nombreuses d'avant en arrière (fig. 5D). Les plaques épimérales sont poilues, à leur bord postérieur comme à leur bord inférieur. L'angle postérieur des plaques 2 et 3 est droit (fig. 5D).

Lobes latéraux de la tête tronqués (fig. 4F); l'oeil allongé et grand.
L'antenne supérieure (fig. 4A) possède un pédoncule grêle, pourvu de soies nettement moins longues que les articles qui les portent. Le premier article pédonculaire porte en plus 2 épines courtes et terminales. Le flagellum principal compte 28 à 40 articles, qui ne portent que peu de sétules courtes. Le flagellum accessoire est composé de 5 ou 6 articles.

L'antenne inférieure (fig. 4B) porte une rangée d'épines sur la face dorsale du 4ème article, tandis que le 5ème article est pourvu de 10 à 12 groupes de longues soies ventrales et médianes. Le flagellum, composé de 14 à 19 articles porte deux rangées de soies très longues sur chacun de ses articles proximaux (figs. 4B, 4C), ce qui lui donnent l'aspect très poilu ayant quelque ressemblance avec la „brosse” trouvée chez $E$. aquilifer. Pas de calcéoles.

Le palpe mandibulaire (fig. 5C) est grêle; le premier article est inerme; le 3ème article porte, ventralement, un ,peigne" d'épines régulier, et distalement 6 longues soies.

La première patte (fig. $4 \mathrm{D}$ ) possède une plaque coxale dont le bord inférieur porte une douzaine de soies relativement longues. Le propode (fig. 4E) porte, sur son bord postérieur, 3 épines auxiliaires („Stiftstacheln”); l'angle palmaire est marqué par 4 épines; ces épines sont nettement séparées par un espace de l'épine palmaire dite médiane, aiguë.

La plaque coxale de la 2ème patte (fig. 5B) est également pourvue de sétules. L'article basal porte, à son bord antérieur, des touffes de soies. Le propode (fig. 6C) est armé, non seulement de „Stiftstacheln" et d'épines de l'angle palmaire, mais également d'une épine médio-palmaire très nettement individualisée.

Chez $P$ 3, et moins nettement chez $P$ 4, les plaques coxales sont poilues, sur leur bord ventral. L'article méral, le carpe et le propode sont très poilus (figs. 6A, 6B).

Les 5èmes, 6èmes et 7èmes pattes (figs. 6D, 5A, 7A) sont à peu près identiques à celles d' $E$. berilloni, quoique l'article basal de P 5 soit légèrement plus svelte que chez berilloni.

La longueur de l'endopode des 3èmes uropodes (fig. 7B) est variable, mais en général cet endopode est court. Très souvent (mais pas toujours) le 2ème article de l'exopode est privé de longues soies.

Le telson (fig. 7C) porte, en dehors des groupes habituels d'éléments subbasaux et terminaux, un groupe de soies dorsal, subterminal. 


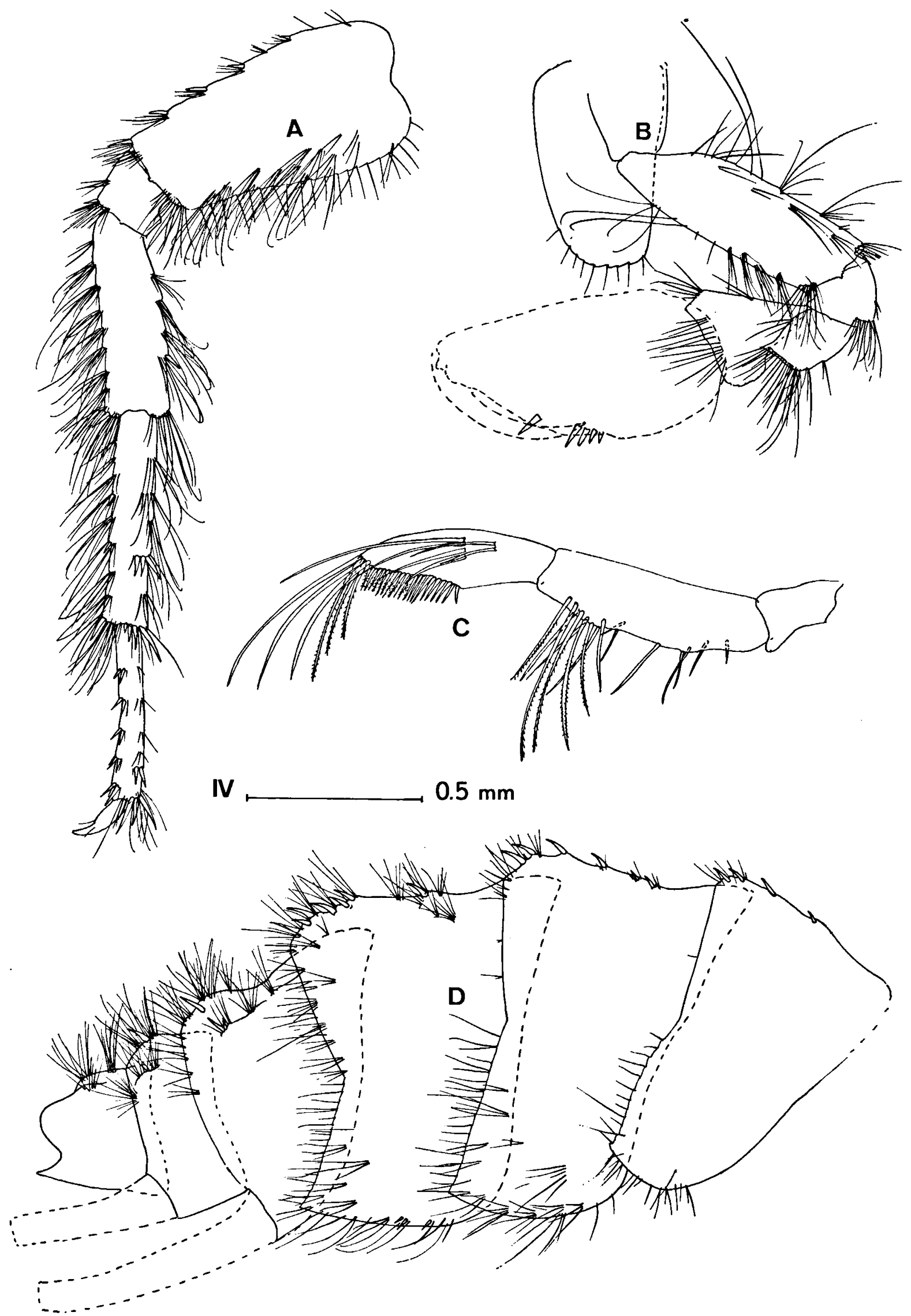

Fig. 5. Echinogammarus zebrinus nov. spec., ô, paratype, de l'Estrigon. A, sixième patte (échelle III); B, deuxième patte (III); C, palpe mandibulaire (IV); D, pléosome et urosome (III). 


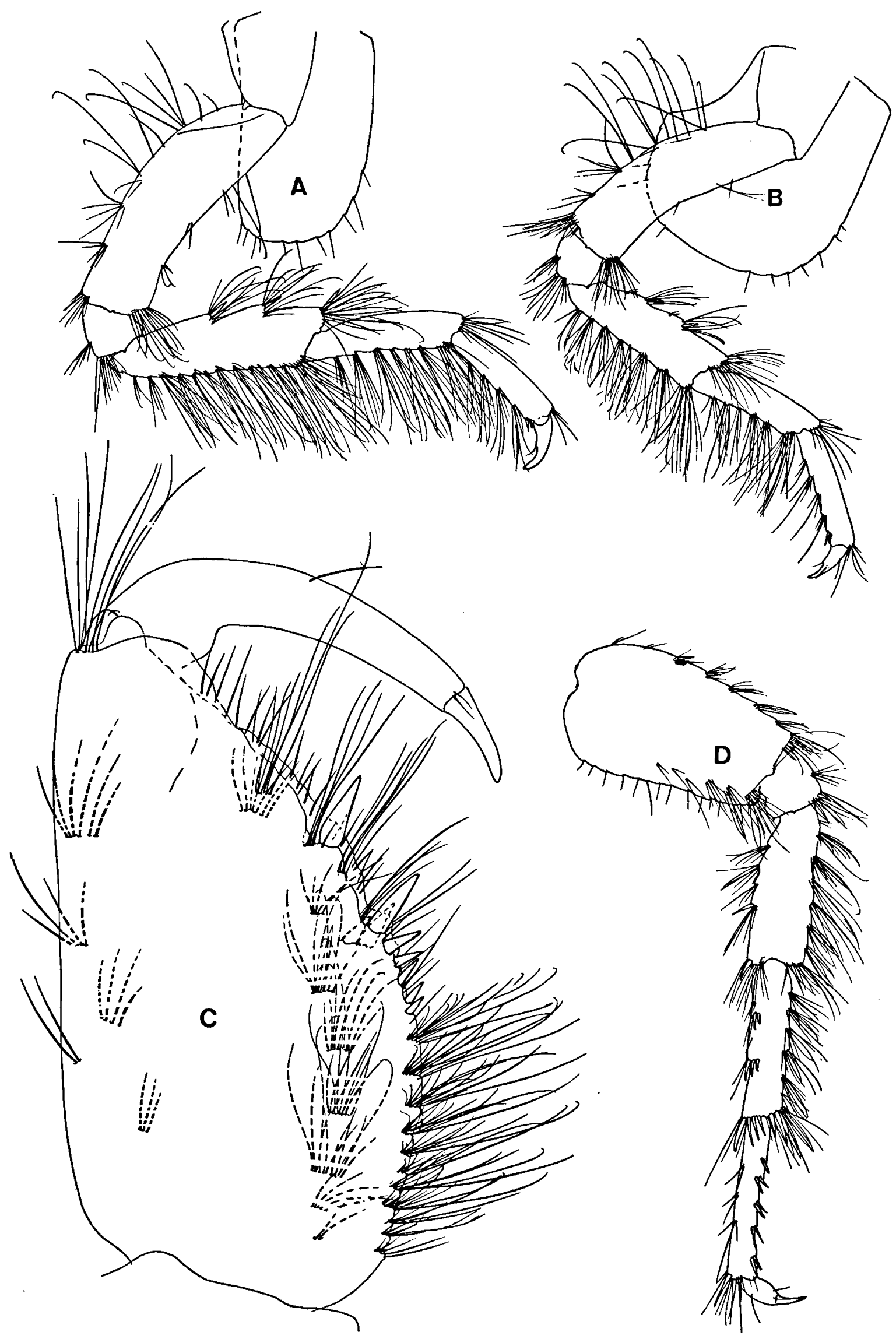

Fig. 6. Echinogammarus zebrinus nov. spec., ô, paratype, de l'Estrigon. A, troisième patte (échelle III); B, quatrième patte (III); C, propode de la deuxième patte (IV); D, cinquième patte (III). Downloaded from Brill.com $04 / 26 / 2023$ 10:00:38AM 


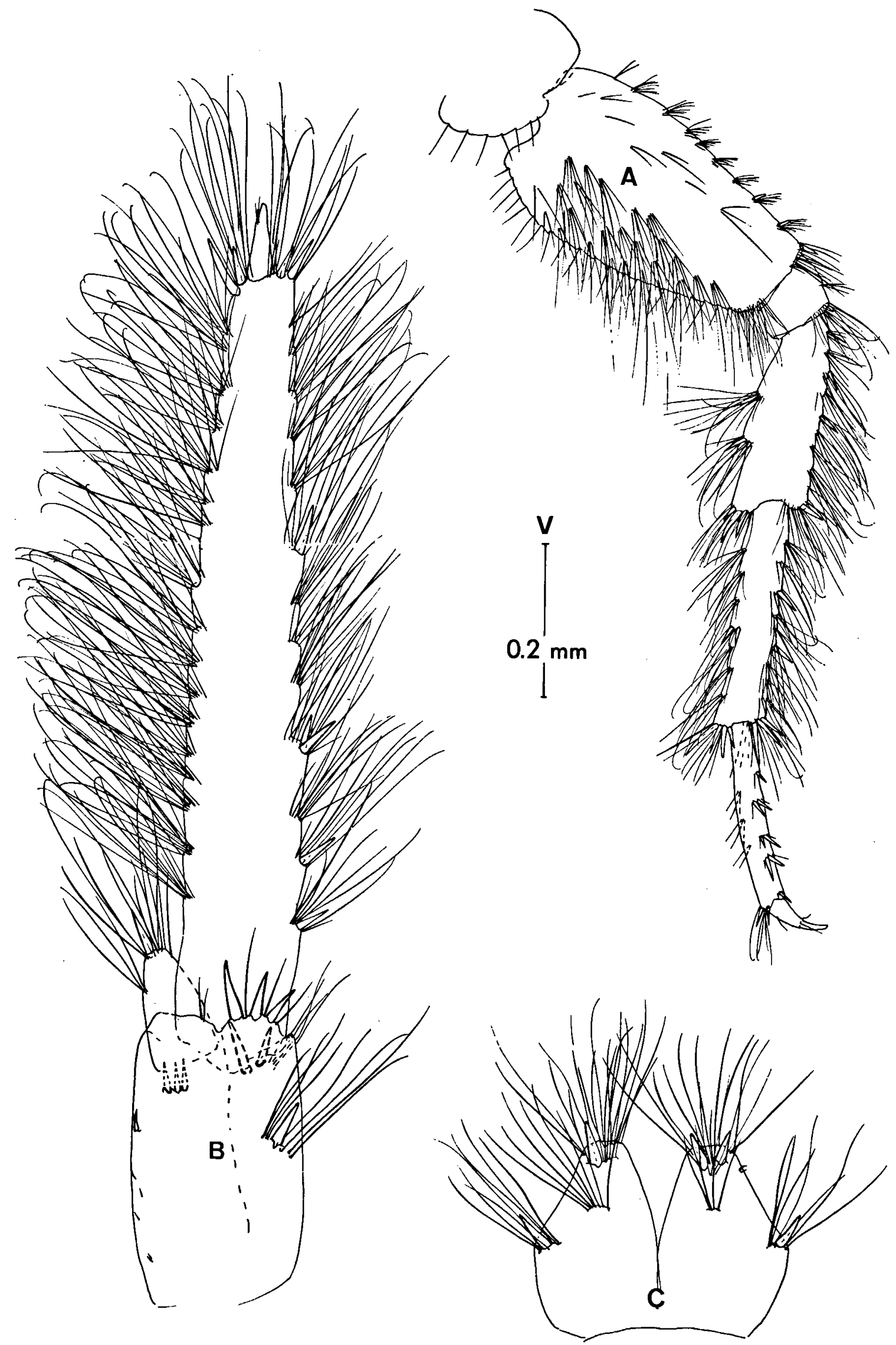

Fig. 7. Echinogammarus zebrinus nov. spec., ô, paratype, de l'Estrigon. A, septième patte (échelle III); B, troisième uropode (IV); C, telson (IV). 


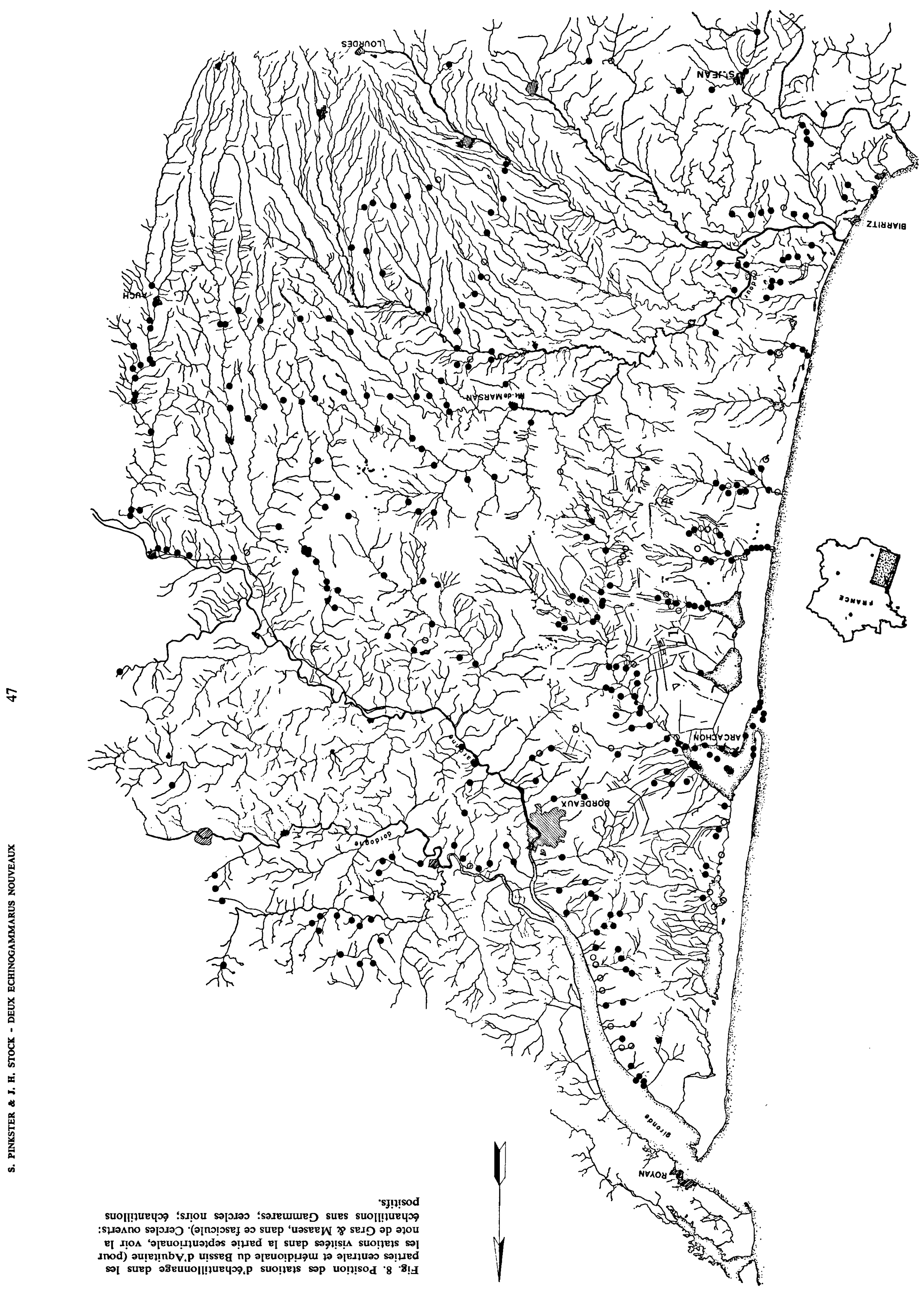


Femelle (planche I fig. 3): Longueur maximale (du bord antérieur de la tête jusqu' à l'extrémité du 3ème uropode) $13 \mathrm{~mm}$. Les pédoncules des antennes supérieures et inférieures sont pourvus de soies plus longues que chez le mâle. Le flagellum de A 2 ne porte pas de „brosse”. Les plaques coxales des pattes 1 à 3 sont, comme chez les mâles, ornementées de soies sur leur bord inférieur. L'article basal de la 7ème patte est moins poilu que chez l'autre sexe, parce que les soies font défaut sur la face interne (mais non sur le bord postérieur). Le groupe de soies dorsal, subterminal, qui caractérise le mâle, manque sur le telson. L'aspect typique - bossu, armé d'épines courbesdu pléosome et de l'urosome est conservé chez la femelle.

Couleur sur le vivant. - Grisâtre, avec des bandes verticales plus foncées sur la tête et sur chaque segment du corps. Ces bandes, très visibles même à l'oeil nu sur le terrain, ont inspirées le nom spécifique proposé pour cette forme nouvelle, zebrinus (voir planche I figs. 2 et 3 ).

Cycle. - Se reproduit apparemment au mois de mai.

Variabilité. - La longueur de l'endopode des 3èmes uropodes peut varier de $10 \%$ à plus de $20 \%$ de la longueur de l'exopode. Si l'endopode est long, il porte 4 à 5 touffes de soies sur son bord interne. Le nombre et la position des épines sur le pléosome et l'urosome sont plus ou moins constants, mais le nombre de soies qui complètent cette armature est très variable; en règle générale les individus les plus gros possèdent plus de soies. Le nombre de touffes de soies sur les articles de pattes 3 à 7 est également variable. Malgré ces caractères, cette espèce ne donne pas l'impression d'être très variable.

Caractères discriminants. - Par l'ensemble de ses caractères, surtout l'armature du dos du pléosome, la présente espèce se classe dans le groupe berilloni du genre Echinogammarus. En France, comme dans toute l'Europe occidentale au nord des Pyrénées, on ne connaît que deux espèces de ce groupe, à savoir $E$. berilloni (Catta, 1878) et $E$. aquilifer Pinkster, 1969 1). La nouvelle espèce combine cer- tains caractères de ces deux espèces avec d'autres qui lui sont propres. Le contour „bossu” du dos, ainsi que son armature d'épines courbes, sont - avec la couleur très distinctive- uniques pour E. zebrinus. Le 5ème article pédonculaire de l'A 2 est plus poilu que chez $E$. aquilifer, tandis que son flagellum est moins poilu que chez aquilifer, mais plus poilu que chez berilloni. Le palpe mandibulaire de la nouvelle espèce porte 6 soies terminales, versus 4 chez aquilifer et 5 chez berilloni. Les plaques coxales 1,2 et 3 portent des soies plus longues chez zebrinus que chez les deux autres espèces. L'épine palmaire médiale des $P$ 2, qui est nettement différenciée chez zebrinus et chez berilloni, fait défaut chez aquilifer. Les pattes 5 à 7 ressemblent à celles de berilloni et sont plus poilues que chez aquilifer. Le groupe dorsal de soies sur le telson du mâle de zebrinus, se retrouve chez aquilifer, mais manque chez berilloni. Les épimères 1 à 3 portent, comme chez berilloni, une riche ornementation en soies, qui la distingue nettement d'aquilifer.

Distribution. - Malgré des recherches intensives sur le terrain, l'espèce nouvelle n'a été trouvée que dans une région très limitée autour de Mont-deMarsan. La figure 8 montre l'ensemble des stations d'échantillonage dans le Bassin d'Aquitaine; l'aire de distribution très restreinte d' $E$. zebrinus, comme la montre le fig. 9, n'est donc pas le résultat d'un échantillonnage insuffisant, mais bien le reflet de la situation naturelle. L'espèce est très abondante à l'intérieur de son aire de distribution où elle habite des eaux dont la dureté varie entre 3,3 et $69,8 \mathrm{mg} \mathrm{Ca} / 1$. Contrairement à la situation observée chez $E$. spinulicornis, dans les stations où il existe, $E$. zebrinus est souvent l'espèce dominante comparée aux autres Gammares. Comme nous l'avons déjà précisé, cette espèce vit dans des eaux dont la dureté est très variable, et, contrairement à la plupart des autres Gammares sa répartition n'est pas liée à la végétation terrestre; en effet, on la rencontre aussi bien dans des ruisseaux bordés par des pins que par des feuillus.

Malgré cette valence écologique élevée, l'espèce n'habite qu'une aire de distribution très restreinte; nous devons admettre que la raison de ce type de distribution nous échappe complètement. 


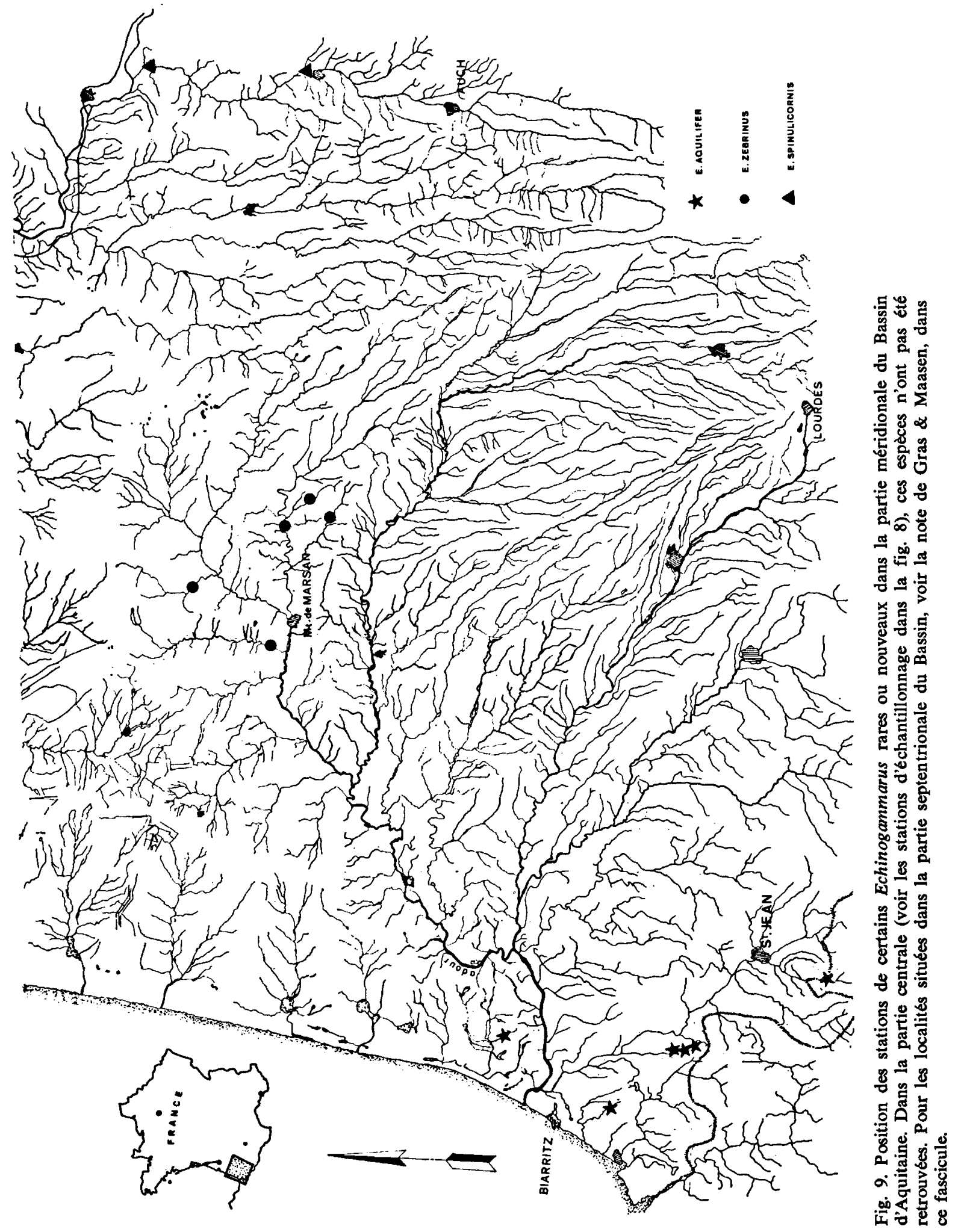




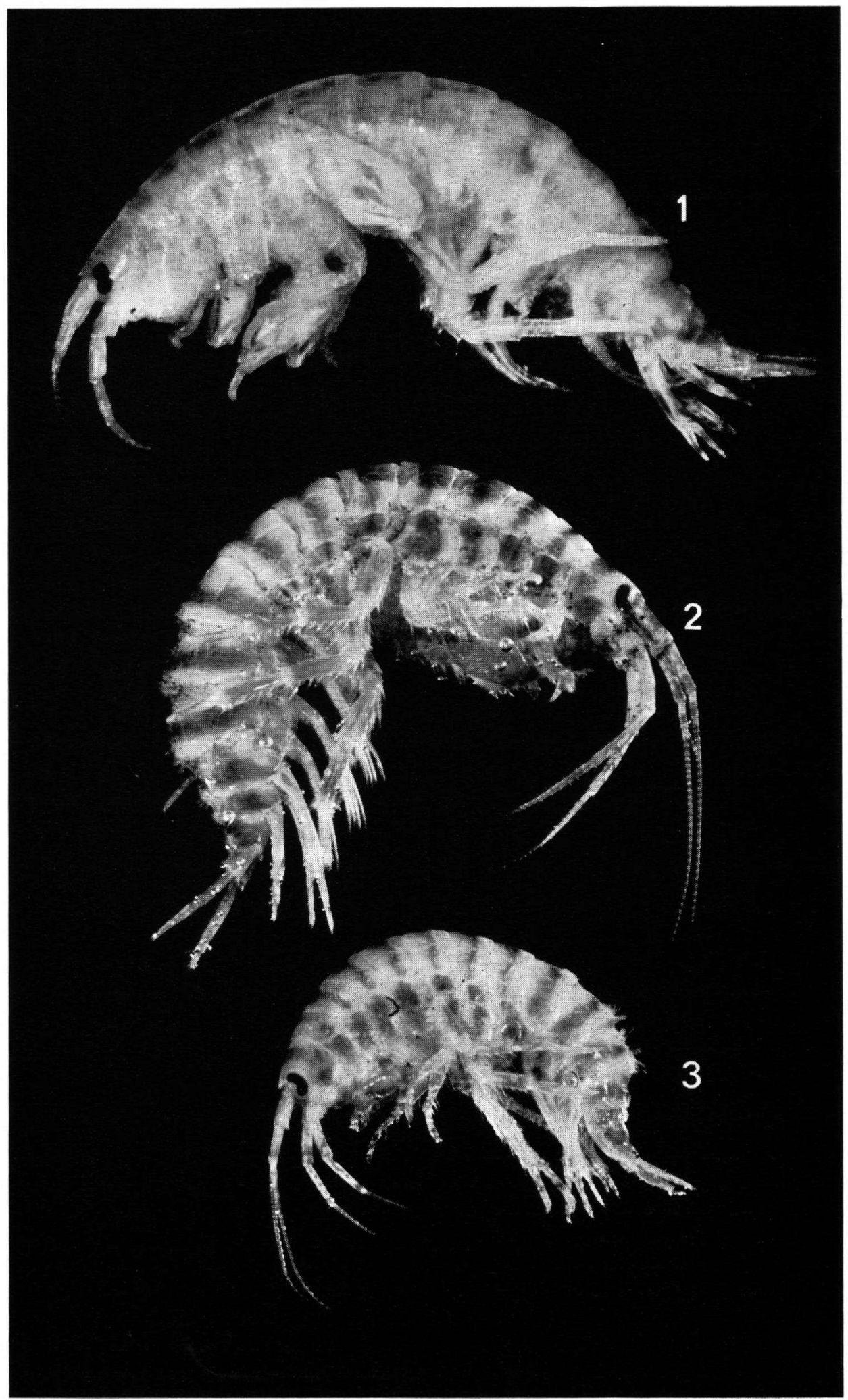

Planche I

1, Echinogammarus spinulicornis nov. spec., to ;

2, Echinogammarus zebrinus nov. spec., ô;

3, Echinogammarus zebrinus nov. spec., $\circ$. 


\section{REFERENCES BIBLIOGRAPHIQUES}

Gras, J. M. J. F. \& A. M. J. MaAsen, 1971. Les Gammaridés des eaux continentales et saumâtres du SudEst de la région armoricaine et du Nord du Bassin d'Aquitaine. Bijdr. Dierk., 41 (1) : 52-60.

Kant, P., W. de Leeuw, S. Pinkster, A. E. Rijnberg \& J. H. Stock, 1968. La répartition d'espèces de Gam. maridae dans quelques étangs au nord de Banyulssur-Mer. Versl. zoöl. Werkexc. Banyuls-sur-Mer, 1968: 3-21 (Zoöl. Mus. Univ. Amsterdam).

Kant, P., S. Pinkster \& J. H. Stock, 1968. Sur la présence en Méditerranée de Pectogammarus planicrurus Reid, 1940. Vie Milieu, (A) 19 (1A) : 207-209.

Pinkster, S., 1969. A new Echinogammarus of the berilloni-group - E. aquilifer nov. sp., from the Pyrenees (Crustacea, Amphipoda). Bull. zool. Mus. Univ. Am- sterdam, 1 (11) : 137-150.

Pinkster, S., A. L. Dennert, B. Stock, J. H. Stock, 1970. The problem of European freshwater populations of Gammarus duebeni Liljeborg, 1852. Bijdr. Dierk., 40 (2) : $116-147$.

STock, J. H., 1967. A revision of the European species of the Gammarus locusta-group (Crustacea, Amphipoda). Zool. Verhand. Leiden, 90 : 1-56.

-, 1968. A revision of the European species of the Echinogammarus pungens-group (Crustacea, Amphipoda). Beaufortia, 16 (211) : 13-78.

STOCK, J. H., H. NiJssen \& P. KANT, 1966. La répartition écologique des Amphipodes de la famille des Gammaridae dans La Slack et son estuaire. Bull. zool. Mus. Univ. Amsterdam, 1 (3) : 19-30.

Reçu: le 15 août 1970 